\title{
HCV Testing and Treatment in a National Sample of US Federally Qualified Health Centers during the Opioid Epidemic
}

\author{
Sabrina A. Assoumou, MD ${ }^{1,2} \mathbb{D}^{\mathrm{D}}$, Jianing Wang, $\mathrm{MSC}^{7}$, Shayla Nolen, $\mathrm{MPH}^{7}$, \\ Golnaz Eftekhari Yazdi, MSC ${ }^{7}$, Kenneth H. Mayer, MD ${ }^{3,4}$, Jon Puro, MPA:HA \\ Joshua A. Salomon, $P h D^{6}$, and Benjamin P. Linas, $M D^{1,2,7}$
}

\begin{abstract}
'Section of Infectious Disease, Department of Medicine, Boston Medical Center, Boston, MA, USA; ${ }^{2}$ Section of Infectious Disease, Department of Medicine, Boston University School of Medicine, Boston, MA, USA; ${ }^{3}$ Fenway Health, The Fenway Institute, Boston, MA, USA; ${ }^{4}$ Beth Israel Deaconess Medical Center, Harvard Medical School, Infectious Diseases, Boston, MA, USA; ${ }^{5} \mathrm{OCHIN}$, Inc., Portland, OR, USA; ${ }^{6}$ Stanford University School of Medicine, Stanford, CA, USA; 'Department of Epidemiology, Boston University School of Public Health, Boston, MA, USA.
\end{abstract}

BACKGROUND: Federally qualified health centers (FQHCs) serve diverse communities in the United States (U.S.) and could function as important venues to diagnose and treat hepatitis $\mathrm{C}$ virus (HCV) infections.

OBJECTIVE: To determine HCV testing proportion and factors associated with treatment initiation, and treatment outcomes in a large sample of FQHCs around the U.S.

DESIGN: Retrospective cohort study using electronic health records of three hundred and forty-one FQHC clinical sites participating in the OCHIN network in 19 U.S. states.

PARTICIPANTS: Adult patients ( $\geq 18$ years of age) seen between January 01, 2012, and June 30, 2017.

MAIN MEASURES: HCV testing proportion, stratified by diagnosis of opioid use disorder (OUD); treatment initiation rates; and sustained virologic response (SVR), defined as undetectable HCV RNA 6 months after treatment initiation.

KEY RESULTS: Of the 1,508,525 patients meeting inclusion criteria, 88,384 (5.9\%) were tested for HCV, and 8694 $(9.8 \%)$ of individuals tested had reactive results. Of the 6357 with HCV RNA testing, 4092 (64.4\%) had detectable RNA. Twelve percent of individuals with chronic HCV and evaluable data initiated treatment. Of those, $87 \%$ reached SVR. Having commercial insurance (aOR, 2.11; 95\% CI, 1.46-3.05), older age (aOR, 1.07; 95\% CI, 1.06-1.09), and being Hispanic/Latino (aOR, 1.87; 95\% CI, 1.38-2.53) or Asian/Pacific Islander (aOR, 2.47; 95\% CI, 1.46-4.19) were independently associated with higher odds of treatment initiation after multivariable adjustment. In contrast, women (aOR, 0.76; 95\% CI, 0.60-0.97) and the uninsured (aOR, 0.15; 95\% CI, 0.09-0.25) were less likely to initiate treatment. Only $8 \%$ of individuals with chronic $\mathrm{HCV}$ were tested for HIV, and 15\% of individuals with identified OUD were tested for HCV.

Electronic supplementary material The online version of this article (https://doi.org/10.1007/s11606-020-05701-9) contains supplementary material, which is available to authorized users.

Received June 19, 2019

Accepted February 3, 2020

Published online March 4, 2020
CONCLUSIONS: Fewer than 20\% of individuals with identified OUD were tested for HCV. SVR was lower than findings in other real-world cohorts. Measures to improve outcomes should be considered with the expansion of $\mathrm{HCV}$ management into community clinics.

KEY WORDS: hepatitis C; health centers; testing; treatment.

$\begin{array}{ll}\text { Abbreviations } & \\ \text { CA } & \text { California } \\ \text { MA } & \text { Massachusetts } \\ \text { OR } & \text { Oregon } \\ \text { US } & \text { United States }\end{array}$

J Gen Intern Med 35(5):1477-83

DOI: $10.1007 / \mathrm{s} 11606-020-05701-9$

(c) Society of General Internal Medicine 2020

\section{INTRODUCTION}

Approximately 3.5 million individuals in the United States (U.S.) are infected with the hepatitis $\mathrm{C}$ virus $(\mathrm{HCV}) .{ }^{1}$ Rates of $\mathrm{HCV}$ infection have been increasing during the opioid epidemic. $^{2-4}$ This surge has been attributed to new cases among young injection drug users. In addition, HCV continues to be underdiagnosed among individuals born between 1945 and 1965 and in persons with a past history of drug use, which in part motivated recent considerations for changes to $\mathrm{HCV}$ testing guidance recommending $\mathrm{HCV}$ testing for all adults aged 18 to 79 years. ${ }^{5,6}$ Federally qualified health centers (FQHCs) care for a diverse patient population predominantly covered by publicly funded health insurance. As FQHCs have been associated with a high burden of HCV infections when compared with the general U.S. population, they could serve as venues to identify and treat HCV-infected individuals. ${ }^{7}$ Little is known about HCV testing and the cascade of HCV care at FQHCs at a time when curative treatment is available with direct acting antivirals (DAAs). ${ }^{8-10}$ Prior data have included clinical trials, commercially insured patients, or small studies at health centers or safety-net hospitals. ${ }^{11-14}$ 
We used the electronic health records (EHR) at OCHIN, one of the largest US FQHC networks, to determine the proportion of $\mathrm{HCV}$ testing, treatment initiation, and sustained virologic response (SVR) or cure. ${ }^{15}$ OCHIN was formerly known as the Oregon Community Health Information Network and it is now referred to as OCHIN after its expansion to other states.

\section{METHODS}

\section{Overview}

We used the OCHIN ${ }^{15}$ EHR to develop a retrospective cohort of individuals seen between January 01, 2012, and June 30, 2017, to determine the proportion of patients with HCV testing, treatment initiation, and SVR, and also evaluated factors associated with each outcome. In addition, we analyzed the proportion of HCV testing among subgroups including individuals born between 1945 and 1965 and patients with identified opioid use disorder (OUD). We also determined the proportion of human immunodeficiency virus (HIV) testing among individuals with chronic $\mathrm{HCV}$.

\section{Sites}

When the data were abstracted, OCHIN included 57 individual FQHCs with 341 clinical sites in 19 US states: Alaska, California, Colorado, Florida, Georgia, Indiana, Massachusetts, Michigan, Minnesota, Montana, Nevada, New Mexico, North Carolina, Ohio, Oregon, Texas, Utah, Wisconsin, and Washington. FQHCs in the U.S. provide the largest source of comprehensive primary health care for underserved populations. More than half of patients seen at OCHIN sites are below the federal poverty level. Approximately half of individuals are on Medicaid, and almost one-fifth do not have health insurance. A third of OCHIN patients identify as Hispanic/Latino and one-fifth as Black/African American. ${ }^{17}$

\section{Outcomes}

We characterized HCV testing trends over time, testing of individuals born between 1945 and 1965, and testing of individuals with documented history of OUD. Injection drug use is the most commonly recorded risk factor for reported $\mathrm{HCV}$ cases. ${ }^{1}$ Recent data show that OUD could be used as a relatively sensitive marker for injection drug use when it is present in the EHR. ${ }^{16}$ We also evaluated the proportion of individuals with chronic HCV (detectable HCV RNA), not already known to be HIV-infected (no previous reactive HIV antibody test), and tested for HIV. We also determined the severity of liver disease at FQHCs during the DAA era.

We determined factors associated with HCV testing, treatment initiation for patients started on "second-generation DAAs and beyond" (Table 5 in Appendix). We used an undetectable viral load 6 months after treatment initiation as a proxy for SVR12, calculated as the proportion of patients with undetectable viral load conditional on treatment completion (defined as having RNA testing 3 months after treatment initiation). A 3-month time frame was selected to capture treatment completion for the majority of patients. We used these estimates as information was abstracted from the EHR and we did not have access to individual charts. We also performed a sensitivity analysis in which SVR was calculated by including individuals with RNA testing any time after 6 months from treatment initiation. This sensitivity analysis excluded all individuals with prescriptions for DAAs, but no RNA tests after treatment initiation.

\section{Study Population}

Inclusion criteria were as follows: (1) at least one visit between January 01, 2012, and June 30, 2017, at a participating OCHIN FQHC; $(2) \geq 18$ years of age.

\section{Data Collection}

Data elements included demographic information and laboratory values (HCV antibody, HCV RNA, HCV genotype, complete blood count including platelet count, and liver function test). We also obtained information on $\mathrm{HCV}$ treatment and clinical visits dates. OCHIN has interfaces with approximately 30 different laboratory entities and testing completed by outside facilities such as Quest or LabCorp is captured in the EHR. Point-of-care testing performed in clinics is also incorporated in the EHR.

\section{Independent Variables}

We included the following covariates in multivariable models of testing, treatment initiation, and treatment outcome: age, gender, race/ethnicity, history of drug use determined by International Classification of Diseases (ICD)-9/10 codes or social history, insurance type, and number of office visits during the time period of interest. We also calculated liver fibrosis stages for individuals with chronic $\mathrm{HCV}$ and for patients initiating $\mathrm{HCV}$ treatment during the time period of interest. For individuals with available information at approximately 12 months from HCV RNA testing, liver fibrosis staging was determined using two alternative non-invasive methods, the aspartate-to-platelet ratio index (APRI) and FIB-4. APRI was calculated with the following formula: ((AST [IU/L]/AST upper limit of normal [IU/L]) / platelets $\left.\left[10^{9} / \mathrm{L}\right]\right) * 100 .{ }^{18}$ We used the following thresholds for liver fibrosis staging: $<0.5$ for F0-F1, $0.5-1.5$ for F2-F3, and $>1.5$ for F4. ${ }^{19}$ FIB-4 was determined with the following formula: age (years) * AST level [U/L]/(platelets $\left.\left[10^{9} / \mathrm{L}\right]\right) *\left(\mathrm{ALT}[\mathrm{U} / \mathrm{L}]^{1 / 2}\right) .{ }^{20}$ We applied the following cutoffs: $<1.45$ for F0-F1, 1.45-3.25 for F2-F3, and > 3.25 for F4.

\section{Statistical Analyses}

We used descriptive statistics to characterize patients seen, and logistic regression to determine factors associated with testing, 
treatment initiation, and SVR. We included significant variables from univariate analysis and confounders in a multivariable model. We also calculated odds ratios (ORs) for treatment initiation, as well as $95 \%$ confidence intervals (CIs). We also performed a sensitivity analysis to determine the possible influence of treatment restrictions according to fibrosis staging (treatment if APRI $\geq 0.5$ corresponding to fibrosis staging greater than F2) for individuals with complete information to calculate APRI scores. All $p$ values were two-sided and we performed statistical analyses using SAS 9.4 (SAS Institute, Cary, NC).

\section{Ethics}

The Boston University Medical Campus Institutional Review Board approved the study.

\section{RESULTS}

The cohort consisted of 1,508,525 patients. The mean age (SD) was 41 (SD 16); $57 \%$ were female. The cohort was 46\% White, 16\% Black/African American, 24\% Hispanic/ Latino, $4 \%$ Asian/Pacific Islander, and 10\% other/unknown (Table 1). Mean and median follow-up time for the cohort were 52 and 48 months, respectively.

\section{Testing Proportions and Trends}

Of the 1,508,525 individuals in the cohort, $6 \%(88,384 /$ $1,508,525)$ had HCV antibody testing results, and $10 \%$ $(8694 / 88,384)$ of those tested had reactive HCV antibody testing (Fig. 1). Seventy-three percent (6357/8694) of HCVseropositive individuals had HCV RNA testing, and of those, 64\% (4092/6357) had detectable HCV RNA suggestive of chronic HCV infection. There was no record of HCV genotype

Table 1 Characteristics for Patients Seen at OCHIN Federally Qualified Health Centers (FQHCs), 2012-2017

\begin{tabular}{|c|c|}
\hline \multirow[t]{2}{*}{ Characteristic } & \multirow{2}{*}{$\begin{array}{l}\text { Total } \\
N(\%) \\
1,508,525\end{array}$} \\
\hline & \\
\hline \multicolumn{2}{|l|}{ Birth cohort } \\
\hline$<1945$ & $98,923(7)$ \\
\hline 1945-1965 & $461,058(30)$ \\
\hline$>1965$ & $948,544(63)$ \\
\hline Female & $861,320(57)$ \\
\hline \multicolumn{2}{|l|}{ Race/ethnicity } \\
\hline White & $724,754(48)$ \\
\hline Black/African American & $250,061(17)$ \\
\hline Hispanic/Latino & $355,840(23)$ \\
\hline American Indian/Alaska Native & $8408(0.6)$ \\
\hline Asian/Pacific Islander & $64,472(4)$ \\
\hline Other/unknown & $104,989(7)$ \\
\hline Any drug use ${ }^{\dagger}$ & $82,590(6)$ \\
\hline Opioid use disorder ${ }^{\dagger}$ & $37,317(3)$ \\
\hline
\end{tabular}

testing for $66 \%$ of individuals with chronic HCV. Of the 1652 individuals with available genotype testing, 66\% (1084/1652) had genotype $1,31 \%(514 / 1652)$ had genotypes $2 / 3$, and $3 \%$ (54/1652) had either genotypes $4 / 6$ or mixed genotypes.

Among the 4092 patients with detectable HCV RNA, 2122 $(52 \%)$ had laboratory results available to estimate liver fibrosis staging by APRI. Forty-five percent of individuals without APRI estimates were missing platelet counts and 35\% lacked ASTs while $32 \%$ had neither ASTs nor platelet counts. Of those with APRI information, approximately $30 \%$ had at least F3 or greater liver fibrosis staging suggestive of advanced disease. Similarly, approximately $25 \%$ had F3 or greater when using FIB-4.

Testing rates increased over time, such that yearly testing for 2016 was three times higher than that for 2012 (Fig. 2 in Appendix). Assuming that the trend observed through the first half of 2017 continued into the second half, we projected that testing for that year would reach approximately four and half times that of 2012. The number of yearly patients seen over the same time period also increased; however; the upward number of tests parallels the rise in patient volume (Fig. 3 in Appendix).

Only $6 \%(27,981 / 461,058)$ of individuals born between 1945 and 1965 were tested for HCV during the time period of interest, and only 14\% of individuals with identified and documented OUD were tested for HCV. Eight percent (335/ 4092) of individuals with chronic HCV were tested for HIV; $8(2 \%)$ were found to have reactive HIV testing. In multivariable logistic regression of the odds of being tested for $\mathrm{HCV}$, being born between 1945 and 1965 (aOR, 1.06; 95\% CI, 1.041.07), Black/African American race/ethnicity (aOR, 1.93; 95\% CI, 1.89-1.96), Hispanic/Latino race/ethnicity (aOR, 1.35; 95\% CI, 1.33-1.38), Asian/Pacific Islander race/ ethnicity (aOR, 1.84; 95\% CI, 1.79-1.90), American Indian/ Alaska race/ethnicity (aOR, 1.24; 95\% CI, 1.13-1.35), other/ unknown race/ethnicity (aOR, 1.54; 95\% CI, 1.50-1.58), and drug use (aOR, 3.07; 95\% CI, 3.01-3.13) were statistically associated with an increased odds of testing; female gender (aOR, 0.76; 95\% CI, 0.75-0.77) was negatively associated with HCV testing (Table 2).

\section{Treatment Initiation}

Of those patients with known HCV viremia, 12\% (489/4092) initiated HCV treatment (Fig. 4 in Appendix). Treatment regimens are listed in Table 5 in Appendix. Treatment initiation for 2016 was 43 times higher than what had been observed for 2012 (Fig. 5 in Appendix). Assuming no change in trend, we projected that initiation in 2017 could have reached approximately 83 times that in 2012 , but the large majority of $\mathrm{HCV}$-infected patients remained untreated.

Of the 489 individuals who were initiated on treatment, 93\% (456/489) had information to determine liver fibrosis staging using APRI. Fifty percent (230/456) had either F0F1, 36\% (163/456) had F2-F3, and 14\% (63/456) had F4. Of note, $30 \%(138 / 456)$ had a least F3 or advanced liver disease. 


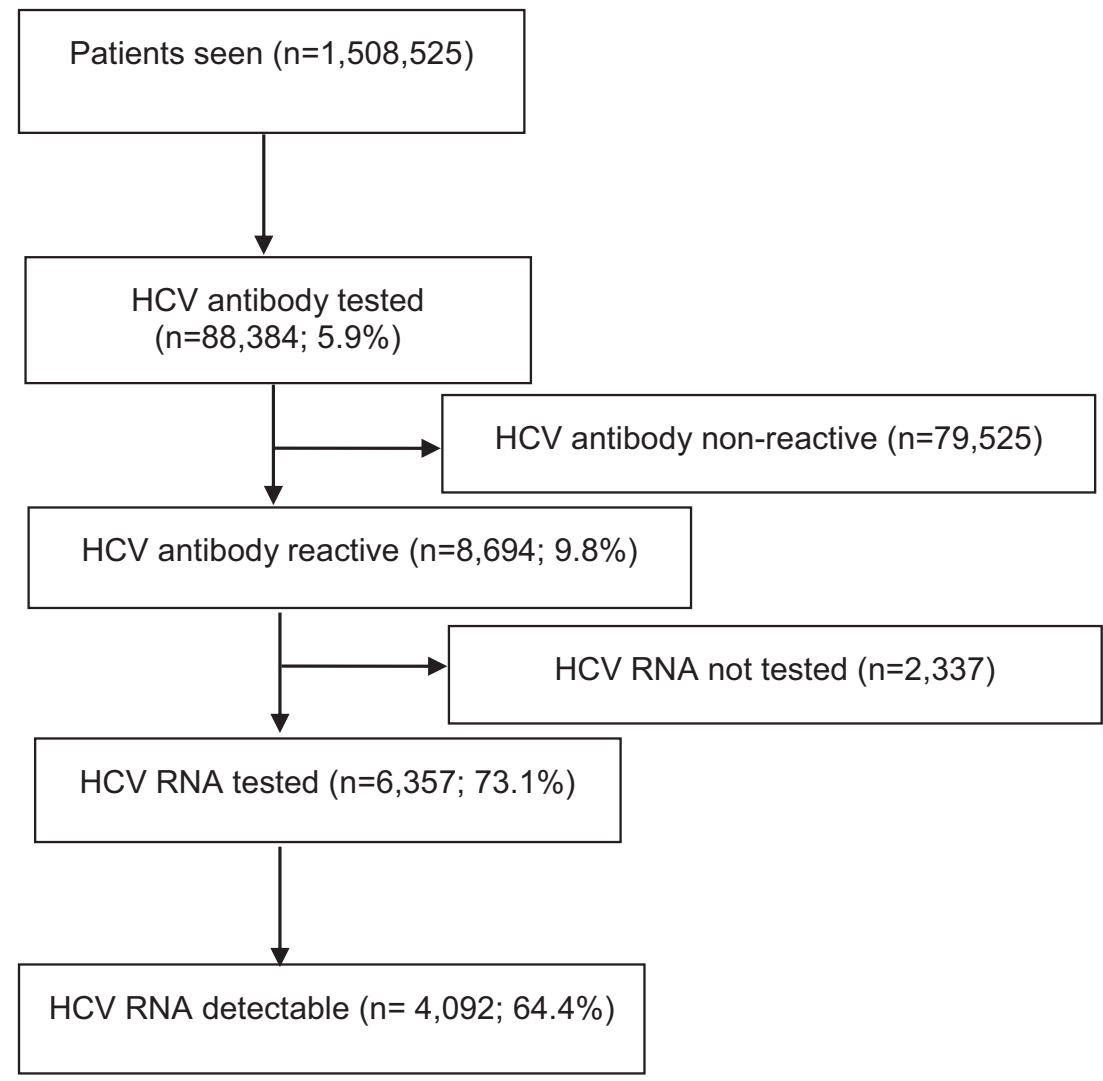

Figure 1 Study flow. FQHC, federally qualified health center; HCV, hepatitis C virus; RNA, ribonucleic acid.

Of the patients initiated on second-generation DAAs $(N=$ 483), 55 did not have any follow-up visits after treatment initiation. When comparing treatment initiation, we found that women (aOR, 0.76; 95\% CI, 0.60-0.97) and the uninsured (aOR, 0.15 ; 95\% CI, 0.09-0.25) were less likely to initiate treatment. In contrast, older patients (aOR, 1.07; 95\% CI, 1.06-1.09), individuals with commercial insurance (aOR, 2.11; 95\% CI, 1.46-3.05), patients who reported Hispanic/
Latino race/ethnicity (aOR, 1.87; 95\% CI, 1.38-2.53), patients who reported Asian race/ethnicity (aOR, 2.47; 95\% CI, 1.464.19), or patients who reported "other" race/ethnicity (aOR, 1.88 ; $95 \% \mathrm{CI}, 1.30-2.70$ ) were more likely to initiate treatment (Table 3). We also determined that fibrosis staging was not associated with treatment initiation in a sensitivity analysis including patients with complete information to calculate APRI scores.

Table 2 Factors Associated with Hepatitis C Virus (HCV) Testing at OCHIN Federally Qualified Health Centers (FQHCs), $2012-2017$ ( $N=$ 88,384)

\begin{tabular}{|c|c|c|c|c|}
\hline Predictor & $\begin{array}{l}\text { HCV tested } \\
N(\%) \\
\mathbf{8 8 , 3 8 4}(6)\end{array}$ & $\begin{array}{l}\text { HCV not tested } \\
N(\%) \\
1,420,141(94)\end{array}$ & $\begin{array}{l}\text { Univariate } \\
\text { Odds ratio }(95 \% \mathrm{CI})\end{array}$ & $\begin{array}{l}\text { Adjusted } \\
\text { Odds ratio (95\% CI) }\end{array}$ \\
\hline \multicolumn{5}{|l|}{ Birth cohort } \\
\hline $1945-1965$ & $27,981(31.7)$ & $433,077(30.5)$ & $1.05(1.04-1.07)$ & $1.06(1.04-1.07)$ \\
\hline$<1945$ or $>1965$ & $60,403(68.3)$ & $987,064(69.5)$ & Reference & Reference \\
\hline \multicolumn{5}{|l|}{ Sex } \\
\hline Male & $44,916(50.8)$ & $602,289(42.4)$ & Reference & Reference \\
\hline Female & $43,468(49.2)$ & $817,852(57.6)$ & $0.71(0.70-0.72)$ & $0.76(0.75-0.77)$ \\
\hline \multicolumn{5}{|l|}{ Race/ethnicity } \\
\hline White & $34,599(39.2)$ & $690,155(48.6)$ & Reference & Reference \\
\hline Black/African American & $21,358(24.2)$ & $228,703(16.1)$ & $1.86(1.83-1.90)$ & $1.93(1.89-1.96)$ \\
\hline Hispanic/Latino & $19,966(22.6)$ & $335,874(23.7)$ & $1.19(1.17-1.21)$ & $1.35(1.33-1.38)$ \\
\hline American Indian/Alaska Native & $538(0.6)$ & $7870(0.6)$ & $1.36(1.25-1.49)$ & $1.24(1.13-1.35)$ \\
\hline Asian/Pacific Islander & $4729(5.4)$ & $59,743(4.2)$ & $1.58(1.52-1.63)$ & $1.84(1.79-1.90)$ \\
\hline Other/unknown & $7193(8.1)$ & $97,796(6.9)$ & $1.47(1.43-1.51)$ & $1.54(1.50-1.58)$ \\
\hline \multicolumn{5}{|l|}{ Drug use } \\
\hline Drug use ${ }^{\dagger}$ & $15,321(17.3)$ & $92,522(6.5)$ & $3.01(2.95-3.07)$ & $3.07(3.01-3.13)$ \\
\hline No drug use & $73,063(82.7)$ & $1,327,619(93.5)$ & Reference & Reference \\
\hline
\end{tabular}

${ }^{\dagger}$ History of drug use was determined by International Classification of Diseases (ICD)-9/10 code on the problem list or in the social history 
Table 3 Factors Associated with Treatment Initiation at OCHIN Federally Qualified Health Centers $(F Q H C s), 2012-2017(N=483)$

\begin{tabular}{|c|c|c|c|c|}
\hline Predictor & $\begin{array}{l}\text { Treatment initiation } \\
N(\%) \\
483(12)\end{array}$ & $\begin{array}{l}\text { No treatment initiation } \\
N(\%) \\
3609(88)\end{array}$ & $\begin{array}{l}\text { Univariate } \\
\text { Odds ratio }(95 \% \mathrm{CI})\end{array}$ & $\begin{array}{l}\text { Adjusted } \\
\text { Odds ratio (95\% CI) }\end{array}$ \\
\hline Age at treatment initiation, mean (SD) & $51(1)$ & $43(12.8)$ & $1.07(1.06-1.08)$ & $1.07(1.06-1.09)$ \\
\hline \multicolumn{5}{|l|}{ Sex } \\
\hline Male & $340(70.1)$ & $2319(64.3)$ & Reference & Reference \\
\hline Female & $143(29.6)$ & $1290(35.7)$ & $0.76(0.62-0.93)$ & $0.76(0.60-0.97)$ \\
\hline \multicolumn{5}{|l|}{ Race/ethnicity } \\
\hline White & $251(51.9)$ & $2110(58.5)$ & Reference & Reference \\
\hline Black/African American & $70(14.5)$ & $632(17.5)$ & $0.93(0.70-1.23)$ & $0.75(0.54-1.02)$ \\
\hline Hispanic/Latino & $83(17.0)$ & $414(11.5)$ & $1.69(1.29-2.21)$ & $1.87(1.38-2.53)$ \\
\hline American Indian/Alaska Native & $2(0.4)$ & $44(1.2)$ & $0.38(0.09-1.58)$ & $0.50(0.11-2.24)$ \\
\hline Asian/Pacific Islander & $24(4.9)$ & $86(2.4)$ & $2.35(1.47-3.76)$ & $2.47(1.46-4.19)$ \\
\hline Other/unknown & $53(10.9)$ & $323(8.9)$ & $1.38(1.00-1.90)$ & $1.88(1.30-2.70)$ \\
\hline \multicolumn{5}{|l|}{ Insurance } \\
\hline Medicaid & $278(61.6)$ & $2075(61.3)$ & Reference & Reference \\
\hline Medicare & $96(21.6)$ & $368(10.9)$ & $1.95(1.51-2.52)$ & $1.07(0.80-1.42)$ \\
\hline Other public & $2(0.4)$ & $41(1.2)$ & $0.36(0.09-1.51)$ & $0.34(0.08-1.47)$ \\
\hline Commercial & $52(11.6)$ & $166(4.9)$ & $2.34(1.67-3.27)$ & $2.11(1.46-3.05)$ \\
\hline Uninsured & $16(3.6)$ & $736(21.7)$ & $0.16(0.10-0.27)$ & $0.15(0.09-0.25)$ \\
\hline \multicolumn{5}{|l|}{ Drug use } \\
\hline Drug use* & $245(50.7)$ & 1933 (53.6) & $0.89(0.74-1.08)$ & $1.22(0.97-1.54)$ \\
\hline No drug use & $238(49.5)$ & $1676(46.4)$ & Reference & Reference \\
\hline Mean number of clinical visits ${ }^{\dagger}$ & 14 & 7 & $1.05(1.04-1.06)$ & $1.05(1.04-1.06)$ \\
\hline
\end{tabular}

SD, standard deviation

This analysis controls for age, gender, racelethnicity, insurance type, history of drug use, and number of clinical visits

*History of drug use was determined by International Classification of Diseases (ICD)-9/10 code on the problem list or in the social history

tNumber of clinical visits during the period evaluated

\section{Sustained Virologic Response}

Of 483 individuals initiated on second-generation DAAs and with evaluable information, 53\% (257/489) had HCV RNA testing at the end of treatment (Fig. 4 in Appendix). Of those, 93\% (239/262) had undetectable viral load. Sixty-four percent (153/239) had HCV RNA results available at least 6 months after treatment initiation and the overall sustained virologic response (SVR) was 87\% (149/171). In multivariable modeling, none of the variables evaluated were independently associated with attaining SVR (Table 4). In a sensitivity analysis, we calculated a $77 \%$ SVR rate when excluding all individuals who did not have any RNA tests after treatment initiation and including all patients with RNA testing any time after 6 months from treatment initiation.

Table 4 Factors Associated with Sustained Virologic Response at OCHIN Federally Qualified Health Centers, 2012-2017 $(N=149)$

\begin{tabular}{|c|c|c|c|c|}
\hline Predictor & $\begin{array}{l}\text { SVR } \\
N(\%) \\
149(87)\end{array}$ & $\begin{array}{l}\text { Failure } \\
N(\%) \\
22(13)\end{array}$ & $\begin{array}{l}\text { Univariate } \\
\text { Odds ratio }(95 \% \mathrm{CI})\end{array}$ & $\begin{array}{l}\text { Adjusted } \\
\text { Odds ratio }(95 \% \mathrm{CI})\end{array}$ \\
\hline Age at RNA test after treatment*, mean (SD) & $51.1(0.7)$ & $50.6(1.0)$ & $1.73(0.99-2.91)$ & $1.74(0.99-3.07)$ \\
\hline \multicolumn{5}{|l|}{ Sex } \\
\hline $\begin{array}{l}\text { Male } \\
\text { Female }\end{array}$ & $112(75.2)$ & $18(81.8)$ & Reference & Reference \\
\hline Female & $37(24.8)$ & $4(18.2)$ & $1.48(0.47-4.67)$ & $1.67(0.50-5.54)$ \\
\hline \multicolumn{5}{|l|}{ Race/ethnicity } \\
\hline White & $77(51.7)$ & $16(72.7)$ & Reference & Reference \\
\hline Black/African American & $28(18.8)$ & $3(13.6)$ & $1.94(0.53-7.17)$ & $1.56(0.4-6.08)$ \\
\hline Hispanic/Latino & $23(15.4)$ & $2(9.1)$ & $2.39(0.51-11.17)$ & $1.85(0.37-9.31)$ \\
\hline American Indian/Alaska Native & $2(1.3)$ & $0(0)$ & $18.4(0.001-99.8)$ & $19.5(0.001-99.1)$ \\
\hline Asian/Pacific Islander & $10(6.7)$ & $0(0)$ & $10.4(0.001-98.9)$ & $15.5(0.001-99.4)$ \\
\hline Other/unknown & $9(6.0)$ & $1(4.6)$ & $1.87(0.22-15.82)$ & $1.69(0.18-15.43)$ \\
\hline \multicolumn{5}{|l|}{ Insurance } \\
\hline Medicaid & $84(56.8)$ & $14(63.6)$ & Reference & Reference \\
\hline Medicare & $39(26.4)$ & $3(13.6)$ & $2.17(0.59-7.98)$ & $2.84(0.70-11.46)$ \\
\hline Commercial & $22(14.9)$ & $4(18.1)$ & $0.92(0.27-3.06)$ & $1.01(0.28-3.67)$ \\
\hline Uninsured & $3(2.0)$ & $1(4.6)$ & $0.5(0.05-5.15)$ & $0.53(0.04-7.92)$ \\
\hline \multicolumn{5}{|l|}{ Drug use } \\
\hline Drug use ${ }^{\dagger}$ & $83(55.7)$ & $14(63.6)$ & $0.72(0.28-1.82)$ & $1.05(0.38-2.94)$ \\
\hline No drug use & $66(44.3)$ & $8(36.4)$ & Reference & Reference \\
\hline Mean number of clinical visits & 19 & 20 & $0.99(0.96-1.02)$ & $1.00(0.98-1.04)$ \\
\hline
\end{tabular}

$S D$, standard deviation; SVR, sustained virologic response

This analysis controls for age after treatment, gender, racelethnicity, and history of drug use, insurance types, and number of clinical visits $* R N A$, ribonucleic acid

tHistory of drug use was determined by International Classification of Diseases (ICD)-9/10 code on the problem list or in the social history Number of clinical visits during the period evaluated 


\section{CONCLUSION}

We used a large, nationally representative US FQHC network to answer three important questions about HCV testing and care for underserved populations during the opioid epidemic: (1) How frequently is $\mathrm{HCV}$ testing occurring at $\mathrm{FQHCs}$ and what are the trends over time?; (2) How many patients are treated during the DAA era and what are the barriers to treatment?; (3) Are cure rates at FQHCs similar to clinical trials and specialty care medical centers? We found that yearly HCV testing increased since the 2012 guidance for one-time testing of individuals born between 1945 and 1965, but those rates are still low. Only approximately 10\% of individuals born between 1945 and 1965 were tested. As we did not have access to testing prior to the period evaluated, it is unclear if some individuals had been tested for HCV before the start of the study. We also found that during the opioid epidemic, only $15 \%$ of individuals with identified OUD were tested for HCV.

We found that approximately 1 in 10 individuals tested for $\mathrm{HCV}$ was seropositive suggesting that $\mathrm{FQHCs}$ are high-yield venues for $\mathrm{HCV}$ testing. Although this number represents a seropositivity rate as opposed to prevalence, it is approximately 10 times the $\mathrm{HCV}$ prevalence found in the general U.S. population. ${ }^{21}$ It is also notable that only $8 \%$ of individuals with chronic $\mathrm{HCV}$ were also tested for HIV. Recent HCV and HIV outbreaks among individuals with a history of injection drug use have reinforced that these two infections need to be actively tested in individuals at risk. ${ }^{22} \mathrm{We}$ also found that approximately one-third of patients with reactive $\mathrm{HCV}$ antibody did not have follow-up confirmatory RNA testing. This finding is similar to other studies in settings without reflex HCV RNA testing ${ }^{13,23}$ and underlines the importance of automatically testing all positive samples for confirmatory testing. In terms of treatment, it is somewhat reassuring that treatment initiation rates increased dramatically over the study period, but even by 2017 , 4 years into the era of DAA therapy for $\mathrm{HCV}$, only a small minority had been treated. Although some patients were likely referred to specialty centers for treatment and those data are not included in this study, it is unlikely that referrals make up the large treatment gap.

Women were less likely to be tested for HCV and initiated on treatment when compared with men. This is notable given that prior data demonstrate that women are more likely to access medical care. ${ }^{24}$ We also found that individuals with commercial insurance were twice as likely as those with public insurance to initiate treatment. The national discussion of HCV treatment includes a debate between cost control and Medicaid treatment restrictions based on both liver fibrosis stage and active drug use. ${ }^{25}$ Medicaid programs in some states have loosened or even removed their formal treatment restrictions, but these data suggest that Medicaid-insured patients may continue to have barriers to therapy.

We identified some potential racial differences in care in that Whites were less likely to initiate treatment when compared with other races/ethnicities. It is possible that individuals identifying as White were more likely to be actively injecting drugs or have unstable social circumstances. ${ }^{26}$ The recent surge in $\mathrm{HCV}$ cases during the opioid epidemic has been among White injection drug users. $^{2}$ Despite HCV treatment guidance recommending treatment of individuals with active injection drug use, it is possible that clinicians might have been less likely to consider them for treatment, or that patients might have been more reluctant to initiate treatment.

In terms of SVR or cure, $87 \%$ of individuals with available information were cured. This was lower than what has been observed in clinical trials, as well as in real-world cohorts of individuals with commercial insurance or in smaller samples of individuals at safety-net hospitals. ${ }^{2-4}$ For example, a large realworld effectiveness study, the Trio Health Study, reported an overall SVR of 94\%, although this cohort also included a large proportion of academic centers. Our findings are similar to a single-site study at an FQHC in Texas where intention-to-treat SVR was approximately $80 \%$ and the TARGET-HCV study, a prospective observational cohort with an overall SVR of $88 \% .{ }^{14} \mathrm{~A}$ variety of studies of primary care-based HCV treatment suggest cure rates similar to those observed in clinical trials. For example, a study in an urban primary care clinic showed a $92 \%$ SVR rate for individuals with available information; however, our findings raise some questions about the need to continuously monitor and improve the effectiveness of $\mathrm{HCV}$ treatment in community-based settings. ${ }^{27}$

There are limitations to our study. This is a retrospective study. We were relying on information in the EHR for treatment initiation. We did not have access to direct pharmacy data and refills. It is also possible that some individuals might have been tested or treated at other facilities, including tertiary centers with access to specialists, which might exaggerate the under-testing and undertreatment seen in this study. Nevertheless, we had access to primary care information on over 1.5 million individuals across 19 states over multiple years and we provide valuable information on treatment outcomes at FQHCs. We did not have information on the proportion of individuals who were referred to outside facilities, but clinicians at $\mathrm{FQHCs}$ included in the analysis reported that the majority of $\mathrm{HCV}$-infected individuals were treated at their primary care facilities. It was estimated that only approximately $5 \%$ of individuals were referred to tertiary care centers. Furthermore, as we determined substance use history by either ICD-9 codes or social history, we were not able to differentiate between individuals who were actively using substances and those with a remote history. According to current guidance, all persons who inject drugs, either remote or currently active, should be evaluated for HCV infection at least once, and persons who are actively using drugs should be tested more frequently. Therefore, our estimate of under-testing among persons with substance use disorders may be conservative and the true gap in testing may be larger. We also did not have information on geographic location for individual sites. Local variations could have impacted referral or insurance patterns. In addition, as we did not have complete information to calculate APRI scores for many patients who were not treated, we were not able to include fibrosis staging in the logistic regression model. Nevertheless, there was no association between fibrosis staging and treatment initiation in a sensitivity analysis including 
only patients with complete information to calculate APRI scores. In addition, it is possible that our study might have missed HIVinfected patients who are in care and who did not have documentation of HIV serology testing in the EHR.

In conclusion, this study demonstrates that FQHCs are not yet reaching their full potential as venues for achieving $\mathrm{HCV}$ elimination goals. We show that testing and treatment have been increasing, but that still only a small proportion of those at highest risk for infection are being evaluated for HCV.

Corresponding Author: Sabrina A. Assoumou, MD; Section of Infectious Disease, Department of Medicine, Boston Medical Center, 801 Massachusetts Ave., Crosstown Center, 2nd Floor, Boston, MA 02118, USA (e-mail: sabrina.assoumou@bmc.org).

Funding Information This project was funded by the U.S. Centers for Disease Control and Prevention, National Center for HIV, Viral Hepatitis, STD, and TB Prevention Epidemiologic and Economic Modeling Agreement (NU38PSO04644). Support was also obtained from the National Institute of Drug Abuse (K23 DA044085, RO1 DA046527, P30 DA040500) and the National Institute of Allergy and Infectious Diseases (P3OAIO42853).

\section{Compliance with Ethical Standards:}

IRB Approval: The Boston University Medical Center Institutional Review Board approved the study.

Conflict of Interest: The authors declare that they do not have a conflict of interest.

Disclaimer: The findings and conclusions of this study are those of the authors and do not necessarily represent the official position of the Centers for Disease Control and Prevention or the National Institutes of Health.

\section{REFERENCES}

1. Centers for Disease Control and Prevention. Surveillance for Viral Hepatitis - United States, 2017. 2017. https://www.cdc.gov/hepatitis/ statistics/2016surveillance/index.htm. Accessed September 18, 2019.

2. Zibbell JE, Iqbal K, Patel RC, Suryaprasad A, Sanders KJ, MooreMoravian $\mathbf{L}$ et al. Increases in hepatitis $\mathrm{C}$ virus infection related to injection drug use among persons aged $\leq 30$ years - Kentucky, Tennessee, Virginia, and West Virginia, 2006-2012. MMWR Morb Mortal Wkly Rep. 2015;64(17):453-458.

3. Zibbell JE, Asher AK, Patel RC, et al. Increases in Acute Hepatitis C Virus Infection Related to a Growing Opioid Epidemic and Associated Injection Drug Use, United States, 2004 to 2014. Am J Public Health. 2017:e1-e7. doi:https://doi.org/10.2105/AJPH.2017.304132

4. Centers for Disease Control and Prevention. Notes from the field: risk factors for hepatitis C virus infections among young adults - Massachusetts, 2010. MMWR Morb Mortal Wkly Rep. 2011;60(42):1457-1458.

5. Smith BD, Morgan RL, Beckett GA, Falck-Ytter Y, Holtzman D, Teo CG et al. Recommendations for the Identification of Chronic Hepatitis C Virus Infection Among Persons Born During 1945-1965. MMWR Recomm Rep. 2012;61(RR-4): 1-32.

6. U.S. Preventive Services Task Force (USPSTF). Draft Recommendation Statement: Hepatitis C Virus Infection in Adolescents and Adults: Screening - US Preventive Services Task Force. https://www.uspreventiveservicestaskforce.org/Page/Document/draft-recommendation-statement/hepatitis-c-screening1. Accessed September 23, 2019.

7. Mayer KH, Crawford P, Dant L, Gillespie S, Singal R, Vandermeer $\mathbf{M}$ et al. HIV and Hepatitis C Virus Screening Practices in a Geographically Diverse Sample of American Community Health Centers. AIDS Patient Care STDS. 2016;30(6):237-246.
8. Kowdley KV, Gordon SC, Reddy KR, Rossaro L, Bernstein DE, Lawitz E et al. Ledipasvir and sofosbuvir for 8 or 12 weeks for chronic HCV without cirrhosis. N Engl J Med. 2014;370(20):1879-1888.

9. Lawitz E, Mangia A, Wyles D, Rodriguez-Torres M, Hassanein T, Gordon SC et al. Sofosbuvir for previously untreated chronic hepatitis C infection. N Engl J Med. 2013;368(20):1878-1887.

10. Guss D, Sherigar J, Rosen P MS. Diagnosis and Management of Hepatitis C Infection in Primary Care Settings. J Gen Intern Med. 2018;33(4):551-557.

11. Tapper EB, Bacon BR, Curry MP, Dieterich DT, Flamm SL, Guest LE et al. Real-world effectiveness for 12 weeks of ledipasvir-sofosbuvir for genotype 1 hepatitis C: the Trio Health study. J Viral Hepat. 2017;24(1):22-27.

12. Younossi ZM, Park H, Gordon SC, Ferguson JR, Ahmed A, Dieterich D et al. Real-World Outcomes of Ledipasvir/Sofosbuvir in Treatment-Naïve Patients With Hepatitis C. Am J Manag Care. 2016;22(6):SP205-11.

13. Assoumou SA, Huang w, Young $\mathbf{K}$, Horsburgh CR LB. Real-world Outcomes of Hepatitis C Treatment during the Interferon-free Era at an Urban Safety-net Hospital. J Heal Care Poor Underserved. 2017;28(4):1333-1344.

14. Alam ZT, Malik H, Hinds CC, Niquette $\mathbf{J}$ AI. Hepatitis C Treatment with Ledipasvir and Sofosbuvir in a Low Socioeconomic Population at a Federally Qualified Health Center [Fqhc]. Gastroenterology. 2018;154(6):S1188-S.

15. Devoe JE SA. The OCHIN community information network: bringing together community health centers, information technology, and data to support a patient-centered medical village. J Am Board Fam Med. 2013;26(3):271-278.

16. Janjua NZ, Islam N, Kuo M, et al. Identifying injection drug use and estimating population size of people who inject drugs using healthcare administrative datasets. Int J Drug Policy. 2018;55:31-39. doi:https:// doi.org/10.1016/j.drugpo.2018.02.001

17. OCHIN. Who We Serve. Facts about Vulnerable Patients. https://ochin. org/vulnerable-patients-the-facts. Accessed September 18, 2019.

18. Lin ZH, Xin YN, Dong $\mathbf{Q J}$, Wang $\mathbf{Q}$, Jiang $\mathbf{X J}$, Zhan SH et al. Performance of the aspartate aminotransferase-to-platelet ratio index for the staging of hepatitis C-related fibrosis: an updated meta-analysis. Hepatology. 2011;53(3):726-736.

19. Chou R WN. Blood Tests to Diagnose Fibrosis or Cirrhosis in Patients With Chronic Hepatitis C Virus Infection. Ann Intern Med. 2013;159(5):372. doi:https://doi.org/10.7326/0003-4819-158-11201306040-00005

20. Vallet-Pichard A, Mallet V, Nalpas B, et al. FIB-4: An inexpensive and accurajte marker of fibrosis in HCV infection. comparison with liver biopsy and fibrotest. Hepatology. 2007;46(1):32-36. doi:https://doi.org/ 10.1002/hep.21669

21. Centers for Disease Control and Prevention. Statistics \& Surveillance | Division of Viral Hepatitis | CDC. Viral Hepatitis - Statistics \& Surveillance. https://www.cdc.gov/hepatitis/statistics/index.htm. Published 2016. Accessed September 18, 2019.

22. Peters PJ, Pontones P, Hoover KW, Patel MR, Galang RR, Shields J et al. HIV Infection Linked to Injection Use of Oxymorphone in Indiana, 2014-2015. N Engl J Med. 2016;375(3):229-239.

23. Vo QT, Onofrey S, Church D, Cranston K, DeMaria A, Klevens R. The Massachusetts Hepatitis C Testing Cascade, 2014-2016. Microbiol Insights. 2019;12:1178636119857961.

24. Centers for Disease Control and Prevention. National Ambulatory Medical Care Survey: 2016 National Summary Tables. https://www.cdc.gov/ nchs/data/ahcd/namcs_summary/2016_namcs_web_tables.pdf. Accessed September 19, 2019.

25. Barua S, Greenwald R, Grebely J, Dore GJ, Swan TT LE. Restrictions for Medicaid Reimbursement of Sofosbuvir for the Treatment of Hepatitis C Virus Infection in the United States. Ann Intern Med. 2015;163(3):215223. doi:https://doi.org/10.7326/M15-0406

26. Suryaprasad AG, White JZ, Xu F, Eichler BA, Hamilton J, Patel A et al. Emerging epidemic of hepatitis $\mathrm{C}$ virus infections among young nonurban persons who inject drugs in the United States, 2006-2012. Clin Infect Dis. 2014;59(10):1411-1419.

27. Lasser K, Heinz A, Battisti L, et al. A Hepatitis C Treatment Program Based in a Safety-Net Hospital Patient-Centered Medical Home. Ann Fam Med. 2017;15(3):258-261. https://www.ncbi.nlm.nih.gov/pmc/articles/ PMC5422088/pdf/0150258.pdf. Accessed September 18, 2019.

Publisher's Note Springer Nature remains neutral with regard to jurisdictional claims in published maps and institutional affiliations. 